Publ. in: Brain Research Reviews ; 67 (2011), 1-2. - S. 331-343

\title{
The role of alpha oscillations in temporal attention
}

\author{
Simon Hanslmayr ${ }^{a, b, *}$, Joachim Gross $^{c, d}$, Wolfgang Klimesch ${ }^{e}$, Kimron L. Shapiro ${ }^{f}$ \\ aUniversity of Konstanz, Department of Psychology, Germany \\ bUniversity of Konstanz, Zukunftskolleg, Germany \\ ${ }^{c}$ University of Glasgow, Institute of Neuroscience and Psychology, UK \\ ${ }^{\mathrm{d}}$ University of Glasgow, School of Psychology, UK \\ eUniversity of Salzburg, Department of Physiological Psychology, Austria \\ ${ }^{\mathrm{f}}$ Bangor University, School of Psychology, UK
}

\section{A R T I C L E I N F O}

Article history:

Accepted 19 April 2011

Available online 28 April 2011

Keywords:

Brain oscillation

Attentional blink

Alpha

MEG

EEG

Resting state

\begin{abstract}
A B S T R A C T
Our brain does not process incoming sensory stimuli mechanistically. Instead the current brain state modulates our reaction to a stimulus. This modulation can be investigated by cognitive paradigms such as the attentional blink, which reveal that identical visual target stimuli are correctly reported only on about half the trials. Support for the notion that the fluctuating state of the brain determines stimulus detection comes from electrophysiological investigations of brain oscillations, which have shown that different parameters of ongoing oscillatory alpha activity $(\sim 10 \mathrm{~Hz})$ can predict whether a visual stimulus will be perceived or not. The present article reviews recent findings on the role of prestimulus alpha oscillatory activity for visual perception and incorporates these results into a neurocognitive model that is able to account for various findings in temporal attention paradigms, specifically the attentional blink.
\end{abstract}

\section{Contents}

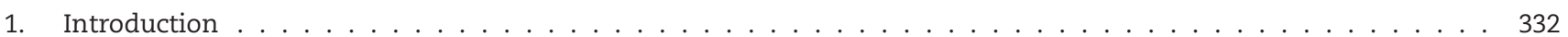

1.1. Externally and internally oriented brain states . . . . . . . . . . . . . . . . . . . . 332

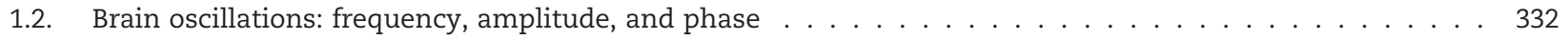

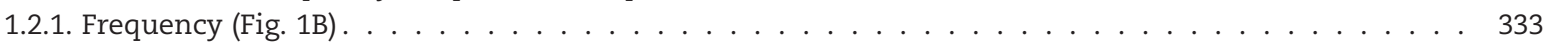

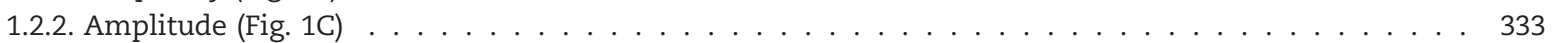

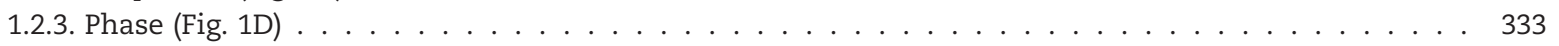

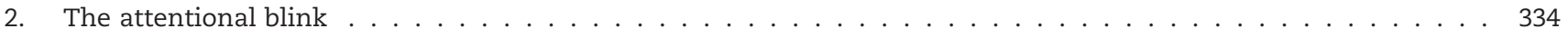

2.1. The AB paradigm and its behavioral outcomes . . . . . . . . . . . . . . . . . . . . . . . 334

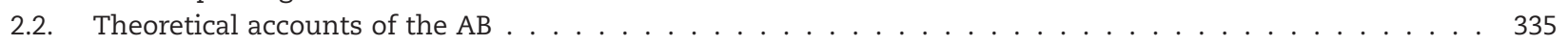

2.3. Brain oscillatory correlates of the $\mathrm{AB} \ldots \ldots \ldots \ldots \ldots$

\footnotetext{
* Corresponding author at: University of Konstanz, Department of Psychology, BOX 23/25, 78457 Konstanz, Germany.

E mail address: simon.hanslmayr@uni konstanz.de (S. Hanslmayr).

URL: http://www.zukunftskolleg.uni konstanz.de/personen/fellows/simon hanslmayr/ (S. Hanslmayr).
}

0165 0173/\$ see front matter @ 2011 Elsevier B.V. All rights reserved. doi:10.1016/j.brainresrev.2011.04.002 
3. Prestimulus alpha activity predicts visual perception $\ldots \ldots \ldots \ldots$

3.1. Alpha amplitude . . . . . . . . . . . . . . . . . . . . . . . . . . . . . . . . 337

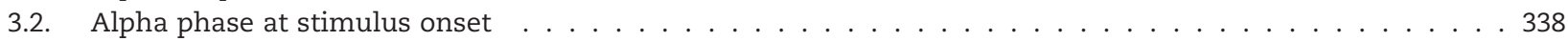

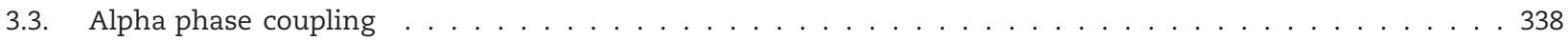

4. Neurophysiological considerations of alpha oscillations . . . . . . . . . . . . . . . . . . 339

4.1. Alpha amplitude and phase at stimulus onset are controlled by thalamo cortical loops . . . . . . . . . . . 339

4.2. Alpha phase coupling reflects cortico cortical communication . . . . . . . . . . . . . . . . . . . 339

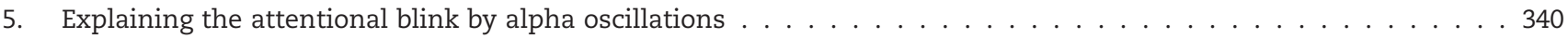

5.1. Alpha amplitudes . . . . . . . . . . . . . . . . . . . . . . . . . . . . . . 340

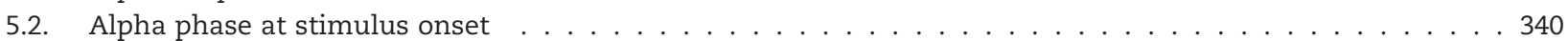

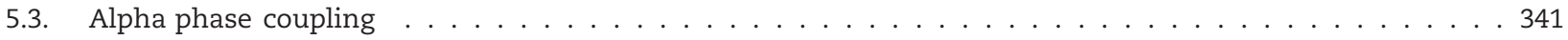

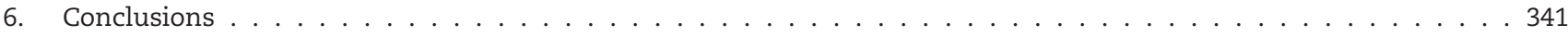

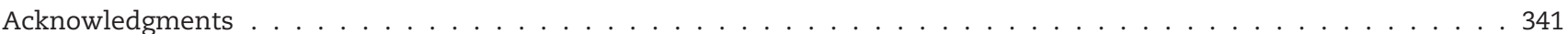

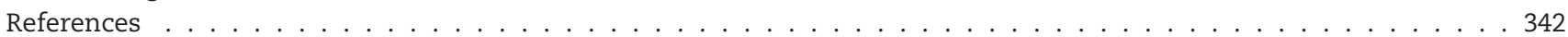

\section{Introduction}

\subsection{Externally and internally oriented brain states}

From time to time we all fail to perceive a salient sensory stimulus because the brain was occupied with some other cognitive process. For instance, we may not recognize an old friend passing by on our way to work, because we are thinking about a new friend who passed by just moments before. Such incidents show that our brain does not process incoming stimuli mechanistically; instead, whether or not a certain stimulus will be perceived partly depends on the brain's current state. Presumably, these brain states differ by whether the brain is biased towards processing stimuli from the external world (the old friend), or towards processing of internal representations (first friend passed). Identifying the neurophys iological signatures of such brain states, and predicting whether or not a sensory stimulus will be perceived, are two of the key goals in modern cognitive neuroscience. Brain oscillations have for a long time been recognized as indexing brain states. Several EEG/MEG studies point toward a funda mental role of ongoing oscillations for shaping perception and cognition. More specifically, a number of recent studies have revealed a significant correlation between the perception of a visual stimulus and different parameters of ongoing alpha activity prior to stimulus presentation ${ }^{1}$ (Ergenoglu et al., 2004; Hanslmayr et al., 2007; van Dijk et al., 2008; Busch et al., 2009; Busch and VanRullen, 2010; Mathewson et al., 2009). These findings suggest that brain oscillations indicate fluctuations between externally and internally oriented brain states, thus determining whether a visual stimulus will be perceived or not.

One of the key paradigms to study the effects of temporal attention is the attentional blink (AB) paradigm (Raymond et

\footnotetext{
${ }^{1}$ To date, the role of prestimulus alpha oscillations on the perception of a sensory stimulus has mostly been explored in the visual domain. Although, similar findings emerged in the somatosensory domain (Linkenkaer Hansen et al., 2004; Schubert et al., 2009), we are not yet aware of any studies in the auditory domain. As most $A B$ studies were also carried out in the visual domain, the current review is focussed on the visual system.
}

al., 1992). The basic finding of the $A B$ is that the processing of a briefly presented stimulus (T1) leads to a significant decrease in the ability to correctly report a subsequent stimulus (T2) if it is presented $\sim 250 \mathrm{~ms}$ after T1. Among others, a prominent hypothesis is that the $A B$ arises because processing the first (external) stimulus (T1) forces the brain into an internal processing mode which renders the system unable to process the second stimulus (T2) to the level of conscious awareness (Shapiro et al., 1997; see Section 2.2 for details). The attentional blink paradigm is thus well suited for studying fluctuations between externally or internally oriented brain states. The goal of the current review is to integrate the findings of the aforementioned EEG/MEG studies into a neurocognitive framework that is able to explain temporal (i.e., sequentially occurring) attentional outcomes, such as revealed by the attentional blink paradigm.

We begin by providing a basic introduction into brain oscillations, with a specific focus on what different parameters of oscillatory activity tell us about the underlying neural processes. In section two, the attentional blink paradigm and its outcomes will be described, focussing on how current cognitive theories explain the $\mathrm{AB}$. In section three, studies which examined the role of prestimulus alpha oscillatory activity for the perception of visual stimuli are reviewed. In the fourth section, the neurophysiological underpinnings of the different parameters of alpha oscillatory activity are consid ered. Finally, in section five a neurocognitive framework is presented that aims to integrate these findings into a coherent account of the $\mathrm{AB}$ and other phenomena of temporal attention.

\subsection{Brain oscillations: frequency, amplitude, and phase}

Brain oscillations reflect rhythmic fluctuations of local field potentials between excitatory and inhibitory states of neural populations. Oscillations are generated by summated excit atory post synaptic potentials (EPSPs) of several thousands of neurons (Lopes da Silva, 1991). The raw EEG/MEG signal contains various different brain oscillations, which can be analyzed by applying spectral analysis (e.g. Wavelet analysis; Fig. 1A). Oscillations are unambiguously characterized by three parameters: (i) frequency (Fig. 1B); (ii) amplitude (Fig. 1C); and (iii) phase (Fig. 1D). 

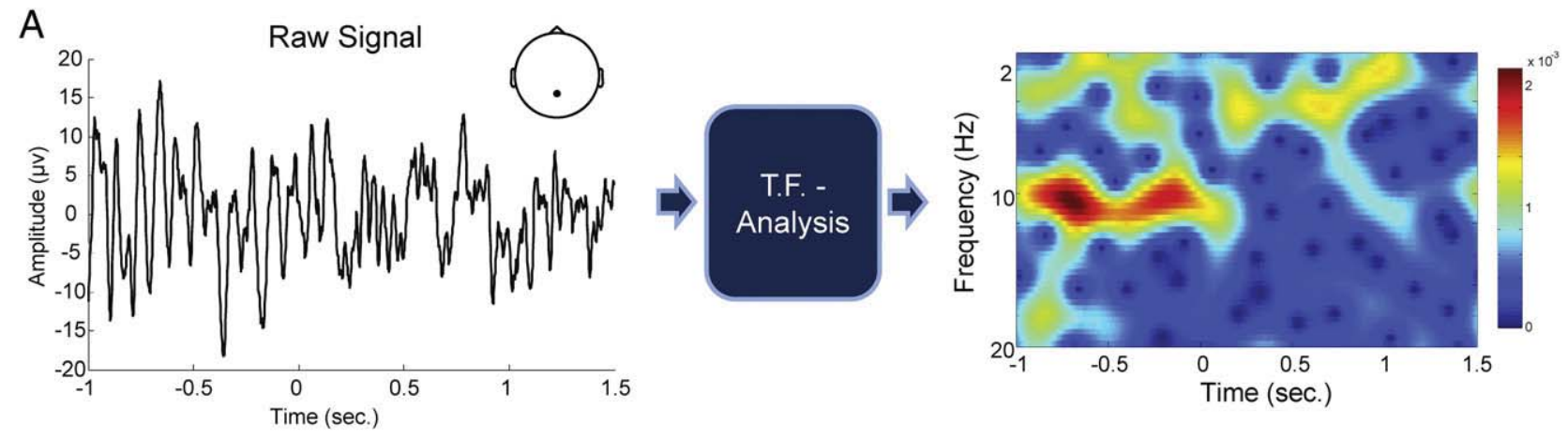

B

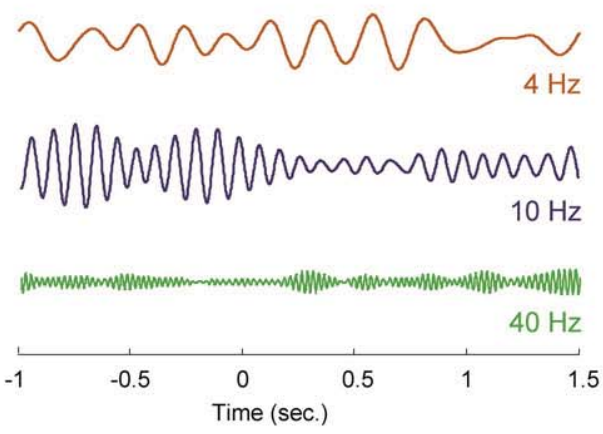

C

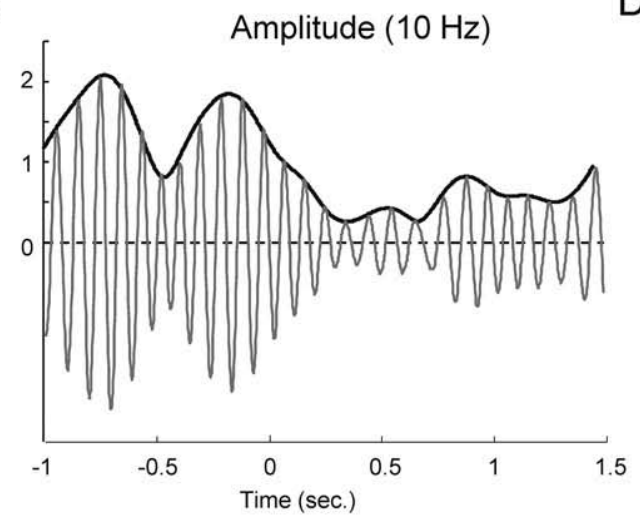

D
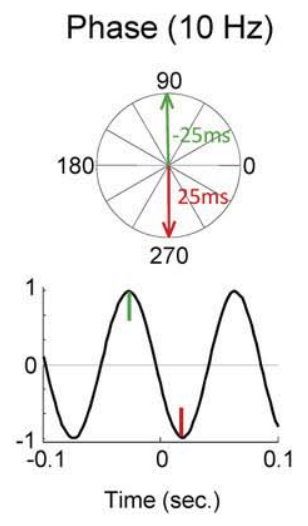

Fig. 1 - Brain oscillations and their parameters. (A) An example of a raw signal as recorded with a parietal EEG electrode is shown on the left. A stimulus was presented at time 0 . The plot on the right shows the results of a time-frequency analysis in which the amplitude is depicted for each time-point ( $x$-axis) and frequency band (y-axis). (B) Theta (4 Hz), alpha (10 Hz), and gamma $(40 \mathrm{~Hz})$ oscillations as extracted from the above raw signal by means of band-pass filtering. (C) The time-course of alpha $(10 \mathrm{~Hz})$ amplitude is shown. Amplitude can be computed from the Hilbert transform of the band-pass filtered signal. (D) Alpha phase at two different time points, ( $25 \mathrm{~ms}$ prior, and 25 after stimulus presentation) is illustrated.

\subsubsection{Frequency (Fig. 1B)}

Different brain networks are hypothesized to oscillate in different frequencies, with small networks oscillating in fast frequencies $(>40 \mathrm{~Hz})$ and large networks oscillating in slower frequencies $(<20 \mathrm{~Hz})$ (von Stein and Sarnthein, 2000; Buzsáki and Draguhn, 2004). Thereby, slower oscillations may repre sent brain networks of a higher hierarchy, encompassing multiple lower level networks, and thereby gating faster oscillations in a top down manner (Lakatos et al., 2005; Lakatos et al., 2008). It is important to note that small and large networks do not refer to the Euclidian space covered by the neural network, but rather refer to the number of connections involved in a network. Thus, a fronto parietal network with monosynaptic connections could be a small network, despite covering a distance of several centimeters.

\subsubsection{Amplitude (Fig. 1C)}

The amplitude of MEG/EEG oscillations is determined by the total number of active neurons and their synchrony, and gives an estimate of how many EPSPs arrive in a given neural assembly at a certain time point (Varela et al., 2001). Therefore, stimulus induced increases and decreases in oscillatory amplitude have been termed event related synchronization and de synchronization, respectively (Pfurtscheller and Ara nibar, 1977; but see Hughes and Crunelli, 2007). Different frequencies, however, behave rather differently in response to sensory stimulation: alpha and beta $(\sim 15 \mathrm{~Hz})$ oscillations typically decrease, whereas theta $(\sim 5 \mathrm{~Hz})$ and gamma $(\sim 40 \mathrm{~Hz})$ oscillations typically increase their amplitudes.

\subsubsection{Phase (Fig. 1D)}

The phase of an oscillation specifies the current position in a given cycle and triggers the timing of neural signals by providing fine grained windows of excitation and inhibition. Evidence for this assumption comes from studies using intracranial recordings in primates and humans which show that the probability of a single neuron to fire is not random with regard to the local field potential, but is triggered by its phase (Lee et al., 2005; Jacobs et al., 2007). Using phase information, two different measures of neural activity can be calculated: (i) phase consistency across single trials, and (ii) phase coupling between EEG/MEG sensors or brain regions. Although these two measures are mathematically closely related, they reflect totally different neural processes and should not be confused. Phase consistency across single trials measures the variability in phase at a certain time point at one 

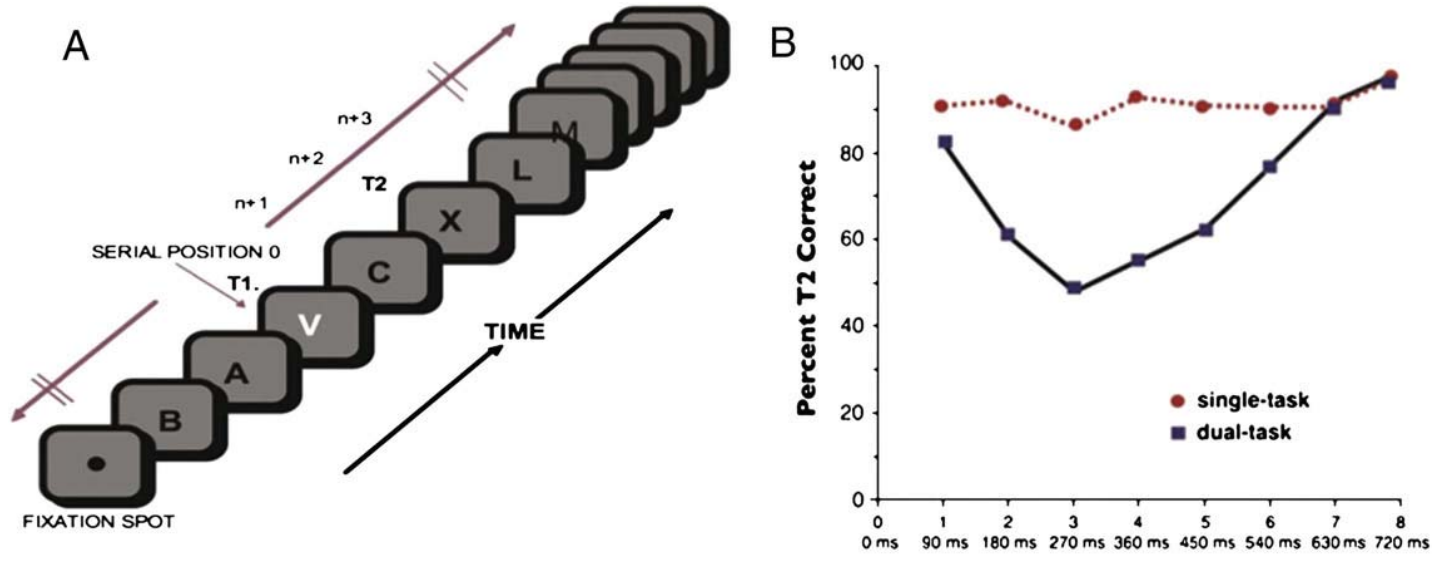

T2 Relative Serial Position

Fig. 2 - The attentional blink paradigm and its typical outcome. (A) A typical trial of an attentional blink experiment is shown. The white letter represents the first target (T1) and the letter ' $X$ ' represents the second target (T2). (B) The typical time-course of the attentional blink is illustrated. Perception performance for T2 is worst at around $270 \mathrm{~ms}$ after T1 presentation.

EEG/MEG sensor, e.g. the $10 \mathrm{~Hz}$ phase at stimulus onset. ${ }^{2}$ Low variability of phase across trials leads to high phase consis tency. Phase coupling between EEG/MEG sensors or brain regions on the other hand, measures the variability of phase differences at a certain time point between two different recording sites. Phase coupling has been interpreted as a measure of communication between distant neural assem blies (Fries, 2005; Varela et al., 2001), and evidence for this assumption has been reported by a recent intracranial study in primates (Canolty et al., 2010). The phase locking value (PLV) is typically used to calculate phase coupling (Lachaux et al., 1999).

Concerning the relationship between ongoing alpha activ ity and the perception of a sensory stimulus three different measures have been explored so far: (i) amplitude, (ii) phase consistency at stimulus onset across single trials, and (iii) phase coupling between distant brain areas. The results of these studies and their implications for understanding the attentional blink are reviewed following a description of this phenomenon.

\section{The attentional blink}

\subsection{The $A B$ paradigm and its behavioral outcomes}

The $A B$ is one of the key approaches used to study the effects of temporal attention. The basic finding of the $A B$ is that processing a briefly presented stimulus (T1) to the level of correct report leads to a dramatic decrease in the ability to correctly report a subsequent stimulus (T2), if T2 is presented between 100 and $500 \mathrm{~ms}$ after T1 (Raymond et al., 1992; Shapiro et al., 1997).

${ }^{2}$ Unfortunately different terms/abbreviations are currently used which all refer to the same measure (Phase locking factor (PLF); Tallon Baudry et al., 1996; Intertrial coherence (ITC); Makeig et al., 2002; Phase locking index (PLI); Gruber et al., 2005).
Although there have been various approaches used to study the AB, the one taken by Raymond et al. (1992) depicts the basic elements of the task and is common to most variants of the paradigm. As depicted in Fig. 2A, a rapid serial visual presentation (RSVP) of stimuli (letters in this example) is presented at approximately 10 items per second $(10 \mathrm{~Hz}$, or alpha frequency band). Each stimulus item is displayed for a brief duration, e.g., $15 \mathrm{~ms}$, with an inter stimulus interval (ISI) of, e.g., $75 \mathrm{~ms}$. Both targets (T1 and T2) are predefined to the subject and are separated by varying SOAs (commonly referred to as 'Lags'), typically ranging from 100 to $800 \mathrm{~ms}$, with the interval between them filled with randomly chosen letter stimuli. In the example in Fig. 2A T1 is defined as being the only white letter in the stream, with the subject required to identify it. In this example, T2 requires a two alternative forced choice judgment as to whether the letter ' $\mathrm{X}$ ' is present or absent. A varying number of stimuli precede the first target so that the subject is not able to predict its occurrence and typically a fixed number of stimuli follow the second target. At least one non target stimulus must follow both T1 and T2, acting in the capacity of a mask. Failure to mask either target results in the absence of an $\mathrm{AB}$. At the end of each RSVP stream, which constitutes a 'trial', subjects are requested to report the identity of $\mathrm{T} 1$ and $\mathrm{T} 2$ in the order in which they were presented. To assess the 'attentional' deficit indexed by the AB as separate and distinct from a 'perceptual' deficit, typically the 'experimental' condition as defined above is compared to a 'control' condition, where the T1 task is not required, though the stimulus stream remains the same.

The typical pattern of results (as shown in Fig. 2B) reveals T2 report to be accurate at Lag $1(\sim 100 \mathrm{~ms})$ and beyond Lag 5 ( $500 \mathrm{~ms})$ but performance at the intervening lags is character istically worse in the experimental condition relative to the control. This interaction between Lag and Condition is the signature of the $\mathrm{AB}$, indicating that attention to $\mathrm{T} 1$ has an effect on T2 performance. Note that T2 performance is assessed only on trials in which $\mathrm{T} 1$ has been reported correctly, as T1 incorrect trials are theoretically indistinguishable from control trials. 


\subsection{Theoretical accounts of the $A B$}

Since the first report of the attentional blink in 1992 there have been a number of theories advanced as to its cause. All theories have in common the basic idea that attending to T1 for subsequent report causes T2 to fail to be encoded and/or retrieved. These accounts all place the attentional blink at a post perceptual level, quite late in the information processing continuum, as evidenced by an early report (Luck et al., 1996) revealing that $\mathrm{T} 2$ is processed to the level of meaning in spite of its inability to be reported correctly on a significant percentage of trials. To briefly present a summary of these accounts, we group them into three categories.

The first category, 'filter' theories, is based on the notion that searching the RSVP stream for a match to the T1 target template (i.e., a white letter in the example above), causes the information processing system to suspend operation for a brief period of time when the match occurs. The suspended processing is argued to prevent interference arising from processing stimuli in the stream subsequent to the T1 target. This account was advanced in the original report by Raymond et al. (1992) and served as the basis for a recent model advanced by Olivers and Meeter (2008), where these investi gators suggest the basis of the $A B$ is a suppression of information processing following the inadvertent processing of the T1 mask. A different variation on filtering was proposed by Di Lollo et al. (2005) and Kawahara et al. (2006) who argued that the $\mathrm{AB}$ occurs when the filter invoked for processing $\mathrm{T} 1$ must be reconfigured to process $\mathrm{T} 2$. It is important to note that this first category of filtering models has in common the notion that the $A B$ arises from a process encountered when the first target is detected with inherently less reliance on processes arising from the occurrence of $\mathrm{T} 2$.

The second category of theories has as their basis the notion that the $\mathrm{AB}$ arises from capacity limitations during the transfer of information to a durable form of memory (i.e., working memory), as required for later retrieval at the end of the trial (cf. Chun and Potter, 1995). Jolicoeur $(1998,1999)$ and Jolicoeur and Dell'Acqua (1998) embellished this notion by connecting it to the psychological refractory period (PRP; Pashler, 1994) phenomenon, arguing that T2 encoding must wait for the completion of critical stages involved in the processing of T1. A recent formal model of the Chun and Potter account has been advanced by Bowman and Wyble (2007) where they proposed that a representation ('type') of T1 and $\mathrm{T} 2$ can be accomplished under conditions producing an $\mathrm{AB}$ but that the episodic ('token') information required to segregate one target from the other cannot occur to T2 while T1 is still being processed. This second category of models is different from the first in that the focus is shifted to an interaction of processes between T1 and T2, though we note that Di Lollo's theory does place a role on the reconfiguration of the filter in response to the occurrence of $\mathrm{T} 2$.

The third category of $A B$ account contends the $A B$ results from a failure to retrieve T2 from working memory, implying that it has successfully been encoded. This account was originally advanced by Shapiro et al. (1994) and later extended by Duncan et al. (1994) and Ward et al. (1996), arguing the two targets and their respective masks compete for retrieval to a stage where they become conscious, i.e., reportable. A related idea has been advanced by Dehaene et al. (2003) who proposed a 'global workspace' model where a target must activate multiple brain areas in a reverberating manner for it to become consciously accessible. This third category of models is differentiated from the previous two by virtue of the emphasis on retrieval, rather than encoding, failure as the basis of the $A B$.

\subsection{Brain oscillatory correlates of the $A B$}

Although much has been learned about the processing of rapidly presented visual information from behavioral investi gations using the $\mathrm{AB}$ paradigm, only relatively recently have there been serious attempts to use neuroscience approaches to understand the brain mechanisms underlying this phe nomenon. To date there have been important findings revealed by the use of fMRI (cf., Marois et al., 2000, 2004; Shapiro et al., 2007) but the slow hemodynamic dependent recovery associated with this approach presents formidable obstacles to real time analysis. Indeed the rapid changes in brain function associated with the rapid information proces sing requirements of the $A B$ paradigm necessitate a different approach. The use of EEG/ERP (e.g., Luck et al., 1996) and, more recently, of MEG (Gross et al., 2004) has stepped in to fill this void. Indeed, to return now to the principle theme of this review as regards the role of ongoing oscillations in the $A B$ phenomenon, we conclude this section with a brief descrip tion of an MEG investigation into oscillatory mechanisms associated with the AB outcome. ${ }^{3}$

Gross et al. (2004; see also Gross et al., 2006) used a variant of the standard $A B$ paradigm, requiring subjects to search for two specified black letters in a stream of black distractor letters. These investigators examined long range synchroni zation between sources of brain activity (cf. Gross et al., 2001). As shown in Fig. 3A, brain oscillations in the lower beta range (15 Hz) were most responding to successful identification of a target (T1 or T2). Source analysis showed that this activity predominantly originated in bilateral frontal, bilateral poste rior parietal, bilateral temporal, and the anterior cingulate and occipital cortical regions (Fig. 3B). As shown in Fig. 3C, the synchronization analysis revealed two different networks, a distractor related and a target related network. Of central interest was to examine the modulation of the target related network with regard to the stimulus elements known to be involved in production of the $\mathrm{AB}$, i.e., $\mathrm{T} 1, \mathrm{~T} 2$, and their respective masks. As shown in Fig. $3 \mathrm{D}$, trials were divided into the four possible types that could arise in the paradigm: distractor only, single target, dual target where T1 but not T2 was identified correctly (i.e., an AB occurred), and dual target where both targets were identified correctly (i.e., no $A B$ occurred). These four trial types were examined for the degree of synchronization modulation (0 1 on the synchronization index, or SI) and de synchronization ( 10 SI) to the four

\footnotetext{
${ }^{3}$ Note that several previous EEG studies also investigated brain oscillatory correlates of the AB (e.g. Keil and Heim, 2009; Keil et al. 2006; Slagter et al., 2009). However, these studies analyzed evoked (i.e. phase locked) activity, and not measures of ongoing brain oscillatory activity (i.e. phase coupling, power) which is the primary focus of this review.
} 


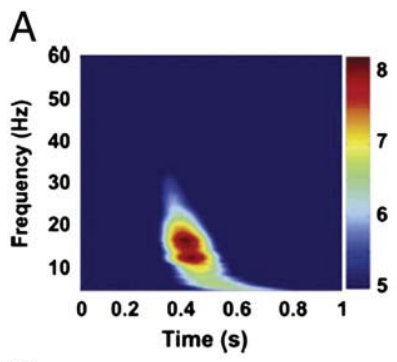

C

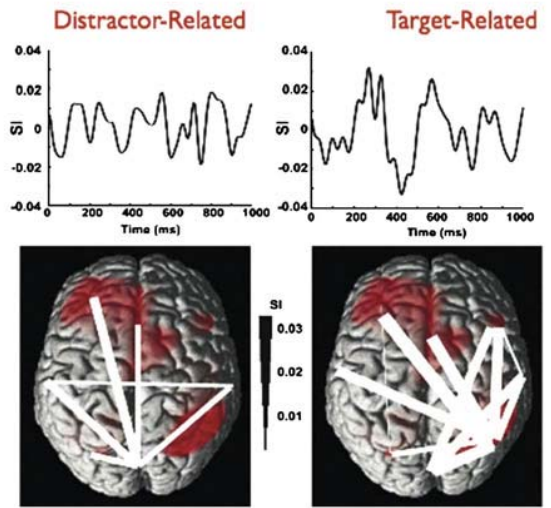

$\mathrm{B}$

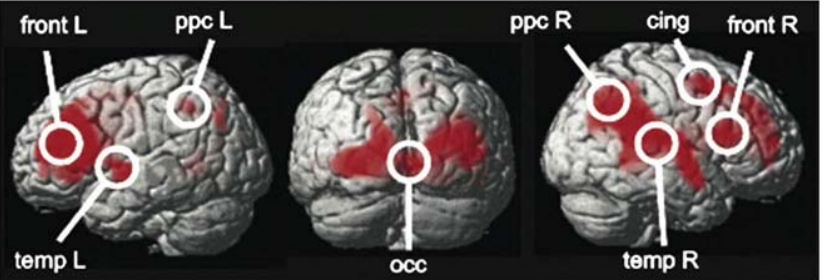

$\mathrm{D}$

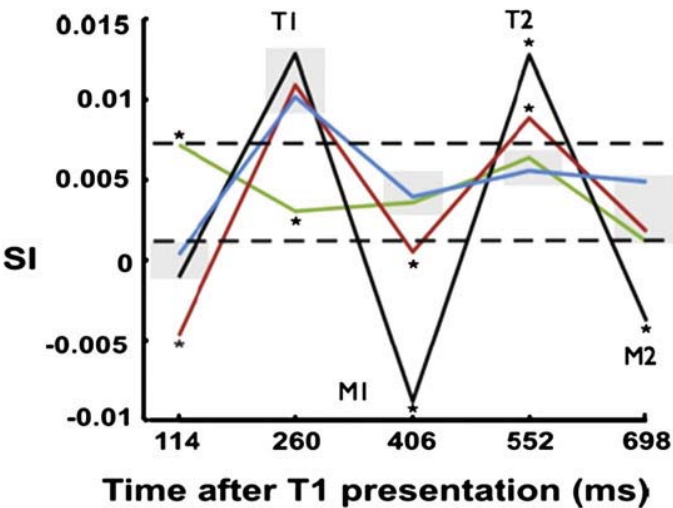

Fig. 3 - (A) Time-frequency representation (TFR) of target-related activity. TFRs timelocked to distractor presentation (at 0 ms) are subtracted from TFRs timelocked to target presentation and averaged across all MEG channels. Power is color-coded and expressed in standard deviations of power at baseline. The TFRs reveal a prominent power increase at around $15 \mathrm{~Hz}$ and 300-500 ms induced by targets. (B) Generators of this component can be identified in occipital and bilateral parietal, temporal and frontal areas (for details of source localization see Gross et al., 2004). (C) Phase synchronization (SI) at $15 \mathrm{~Hz}$ between all combinations of areas allows identification of two networks. One network shows similar modulation of synchronization to each stimulus (distractor and target, left panel) whereas the other network shows a pronounced modulation of synchronization to targets only (right panel). (D) Mean phase synchronization (SI) in the target-related network is shown for four conditions (black: both targets perceived, red: second target not perceived (AB), blue: only T1 was presented, green: no target was presented) in response to 5 stimuli around the first and second targets (T1: first target, M1: first mask, T2: second target, M2: second mask). Note the significant differences between the black line and the red line at the time of the first mask (M1), prior to the second target (T2).

Figure modified from Gross et al., 2004; copyright 2004 National Academy of Sciences, USA.

stimulus elements defined above, i.e., T1, T2, and the T1/T2 masks.

The results (Fig. 3D) reveal an equal amount of synchroni zation occurring whenever a target (T1 or T2) was identified correctly, suggesting synchronization reflects a brain behav ior correspondence to correctly identified targets. Unexpect edly, and of considerable interest, was the de synchronization revealed on no $A B$ trials prior to the second target processing, presumably reflecting inhibition of the mask following $\mathrm{T} 1$. This was interpreted by Gross et al. to suggest that only when the deleterious effect of the mask is uncoupled from the target preceding it (i.e., via de synchronization), can accurate target identification occur. As outlined below, this finding comple ments results from recent EEG studies (Hanslmayr et al., 2007; Kranczioch et al., 2007) showing that reduced long range synchrony in the prestimulus interval predicts successful stimulus identification. In summary, rapidly changing pat terns of synchronization and de synchronization were revealed to index the behavioral outcomes found in the attentional blink paradigm. Specifically relevant for this review is the finding that the synchronization pattern before T2 processing predicted whether or not an $A B$ occurred.

An open question, however, is how these effects, which were found in the lower beta band, relate to alpha oscillations. One possibility would be that the frequency in which the $A B$ effects are found, is related to the rate at which the RSVP stream is presented. In the study by Gross et al. (2004) the stimuli were presented at a rate of $\sim 7 \mathrm{~Hz}$, of which $15 \mathrm{~Hz}$ is very close to the first harmonic $(14 \mathrm{~Hz})$. A recent $A B$ study by Kranczioch et al. (2007) presented their stimuli at a rate of $10 \mathrm{~Hz}$ and replicated the basic finding of Gross et al., in showing that decreased synchronization prior to the second target correlated with its perception. Interestingly, these effects emerged in the alpha frequency band (at $10 \mathrm{~Hz}$ ), which might suggest that stimulation frequency partly de termines the frequency band in which the $A B$ effects occur. Moreover, in EEG/MEG data alpha and beta oscillations very often go hand in hand and it is hard to draw a clear line between upper alpha and lower beta. Likely these questions will be resolved by future studies. 


\section{Prestimulus alpha activity predicts visual perception}

As mentioned above, three different parameters of prestimu lus alpha oscillatory activity have been used successfully in several prior studies to predict the perception of a visual stimulus, (i) amplitude, (ii) phase at stimulus onset, and (iii) phase coupling. The results of these studies are reviewed below.

\subsection{Alpha amplitude}

Alpha amplitudes are most evident in a resting condition prior to the presentation of a stimulus and typically decrease when a sensory stimulus is presented. Ongoing alpha amplitudes, however, do not stay at a consistently high level, but show a significant amount of variation. In continuous EEG/MEG recordings alpha oscillations typically exhibit a pattern of waxing and waning (Fig. 4A; Lopes da Silva, 1991). These characteristics led researchers to conclude that fluctuations in alpha amplitude point to different behaviorally relevant brain states (Linkenkaer Hansen et al., 2001). In line with this assumption recent studies reported that whether or not a visual stimulus will be perceived can be predicted on a trial by trial basis by prestimulus alpha amplitudes (Ergenoglu et al., 2004; van Dijk et al., 2008). In both studies visual stimuli were presented for a very short duration $(3.3 \mathrm{~ms}$ and $16 \mathrm{~ms}$, respectively), close to the sensory threshold, and not masked. Both studies revealed highly similar results in showing a negative correlation between alpha amplitude and perception performance. In both studies periods of high prestimulus alpha amplitude predicted a miss, whereas low alpha amplitude predicted a hit. This effect was most evident over parieto occipital regions, and van Dijk et al. (2008) were able to localize these effects to the precuneus ( BA 7). Using transcranial magnetic stimulation (TMS) similar findings emerged. When TMS is applied over the visual cortex, subjects usually perceive an unstructured flash of light, termed phosphene. Romei et al. (2008) demonstrated that whether or not a phosphene is perceived depends on alpha amplitudes, with low amplitudes predicting phosphene perception. On a

A

$\alpha$ Amplitude
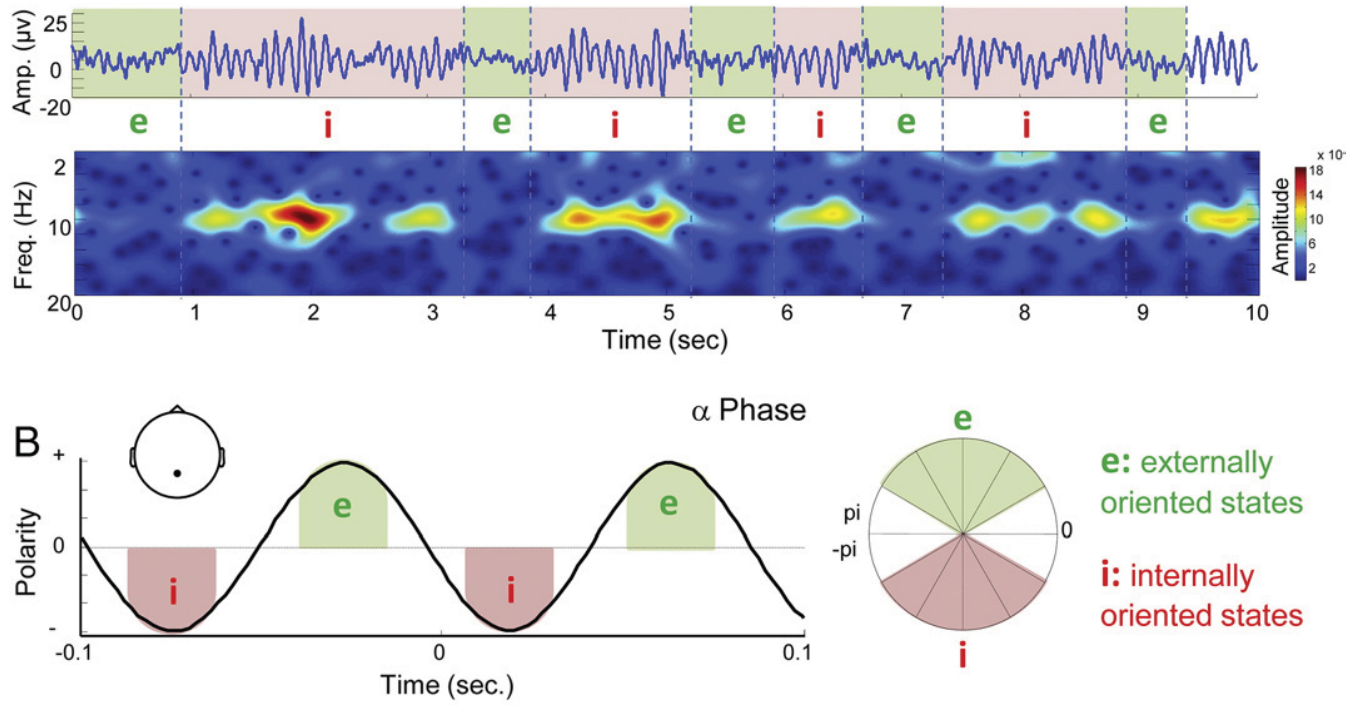

\section{e: externally} oriented states

i: internally oriented states

C $\alpha$ Phase - Coupling

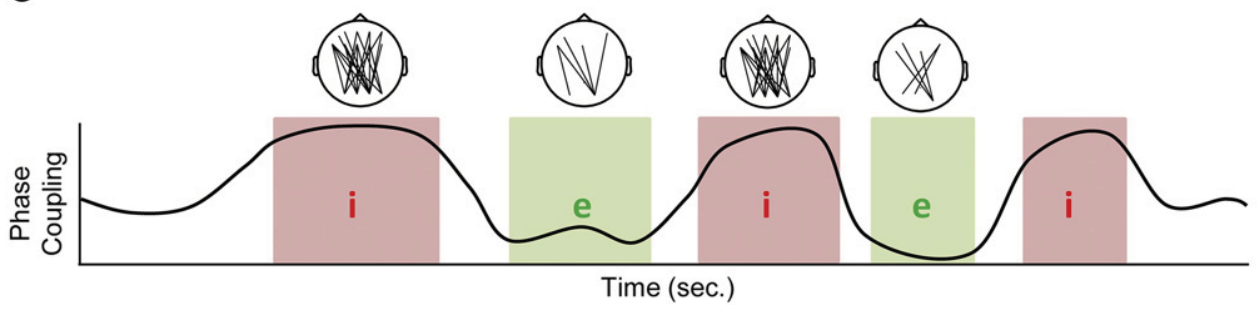

Fig. 4 - Three different parameters of alpha oscillatory activity indicate externally (e) and internally oriented (i) states. (A) The raw signal (upper panel) and time-frequency amplitude (lower panel) of a resting state EEG are shown for a parietal electrode. Note the waxing and waning pattern of alpha activity $(10 \mathrm{~Hz})$. Periods with low alpha amplitude indicate externally oriented states (green), whereas periods with high alpha amplitude indicate internally oriented states (red). (B) Alpha phase and its relation to externally (green) and internally (red) oriented states are shown. Peaks (positive polarity) indicate externally, and troughs (negative polarity) indicate internally oriented states. (C) Alpha phase-coupling and how it indicates externally and internally oriented states are illustrated. High periods of phase-coupling indicate internally oriented states (red), whereas low levels of phase-coupling indicate externally oriented states (green). 
between subjects level, comparable results were reported by Hanslmayr et al. $(2007,2005)$ who showed that subjects exhibiting poor performance in a visual perception task were characterized by high parieto occipital alpha amplitudes.

The above cited studies investigated the role of prestimu lus alpha amplitude utilizing a correlational approach, con trasting trials where the stimulus was perceived with trials where the stimulus was not perceived. Recently, a few studies have emerged that manipulated performance in a perception task by experimentally inducing states of high or low prestimulus alpha amplitude. Romei et al. (2010), for instance, employed repetitive TMS (rTMS) and were able to show that this type of stimulation applied at alpha frequency (and not in other frequencies) induced a decrease in visual perception performance (Romei et al., 2010). Another study used mood induction to manipulate prestimulus alpha amplitudes (Kuh bandner et al., 2009). Based on previous reports showing that mood is quite effective at modulating visual perception, with negative mood enhancing and positive mood decreasing performance (Phelps et al., 2006), Kuhbandner et al. reported that negative mood decreased, whereas positive mood in creased, prestimulus alpha amplitude. Consistently, visual perception performance was better in the negative compared to the positive mood condition. These studies are a first hint that the relationship between prestimulus alpha amplitudes and perception is not a correlative but a causal one.

Taken together, the above reported findings provide strong evidence that alpha amplitude indicates different brain states, which have a bias either toward external or internal proces sing. Thereby, high alpha amplitudes may indicate internally oriented brain states, which make it hard for a briefly presented stimulus to be perceived; low alpha amplitudes may indicate externally oriented brain states, which bias the system toward processing information from the sensory channels (see Fig. 4A). In accordance with two recently proposed theories (Klimesch et al., 2007; Jensen and Mazaheri, 2010), the results suggest that parieto occipital alpha ampli tudes reflect the excitatory/inhibitory state of visual proces sing brain regions, thereby enhancing or diminishing, respectively, the likelihood of stimulus perception.

\subsection{Alpha phase at stimulus onset}

As mentioned above, the phase of an oscillation represents fine grained temporal windows of excitation and inhibition. It has been suggested that alpha oscillation in particular represents time frames of perception (Varela et al., 1981; VanRullen et al., 2006). It is thus reasonable to link the phase of alpha oscillations to the perceptual fate of a visual stimulus. Although a few recent studies have investigated this issue (Busch et al., 2009; Busch and VanRullen, 2010; Mathewson et al., 2009), the same idea was investigated far earlier (Varela et al., 1981; Callaway and Yeager, 1960; Dustman and Beck, 1965). For instance, Callaway and Yeager (1960) showed that faster responses to visual stimuli occur if the stimulus is presented at the positive phase of an alpha cycle (see Dustman and Beck, 1965; for similar results). Varela et al. (1981) presented two stimuli successively, with a very short interval in between, and showed that the subjects perceived the two stimuli as one, if they were presented at the positive peak of the alpha cycle.
Recently, Mathewson et al. (2009) reported comparable results by demonstrating that briefly flashed visual stimuli were more likely to be perceived when presented at the positive than when presented at the negative peak of the alpha phase. Interestingly, Mathewson et al. found that alpha phase predicted perception performance only in those trials where alpha amplitude was high. Thus, alpha phase and amplitude, though being mathematically independent measures, appear not to be empirically independent.

Similar results were reported by Busch et al. (2009) and Busch and VanRullen (2010), although their findings differ from the other studies in several ways: (i) Busch et al. found that alpha phase over fronto central electrodes predicted perception performance, whereas other investigators (Math ewson et al., 2009; Varela et al., 1981; Callaway and Yeager, 1960) reported a parieto occipital topography. (ii) In Busch et al's studies the effects were more pronounced around $7 \mathrm{~Hz}$, at the crossover between theta and alpha frequency bands; not in the classical alpha band, around $10 \mathrm{~Hz}$. (iii) Busch et al. failed to replicate the finding that the positive peak of the alpha cycle is optimal for perception performance, instead the phase of optimal perceptual performance varied considerably across subjects.

To our knowledge, only one study so far was designed to experimentally manipulate alpha phase at stimulus onset (Mathewson et al., 2010). In their study, Mathewson et al. presented a stream of stimuli flickering at $12 \mathrm{~Hz}$, presumably inducing steady state visual evoked potentials in the alpha frequency range. A critical stimulus that had to be detected was presented at a variable interstimulus interval after the prestimulus flicker $(32,59,82,107$, or $130 \mathrm{~ms})$. The results showed that perception performance was best if the stimulus was presented at $82 \mathrm{~ms}$, which exactly matches the period length of $12 \mathrm{~Hz}{ }^{4}$ Although Mathewson et al. (2010) did not measure the EEG, and thus cannot directly establish a link between alpha phase at stimulus onset and perception performance, their results are a first hint that the detection of a visual stimulus can be optimized by manipulating alpha phase at stimulus onset via steady state visual evoked potentials.

Despite the above reported discrepancies all studies come to the common conclusion that the phase of alpha oscillations represents temporally fine grained windows of internally or externally oriented brain states. The majority of these studies indicate that positive phases of EEG alpha oscillations over parieto occipital regions indicate externally, whereas negative phases indicate internally oriented brain states (see Fig. 4B).

\subsection{Alpha phase-coupling}

Whereas several studies have explored how prestimulus alpha amplitude and phase at stimulus onset influence the perceptual fate of a visual stimulus, far fewer studies have investigated the role of prestimulus phase coupling. One reason might be the extensive use of computational power that is required for calculating phase coupling due to the many possible pairs of recording sites. For example a standard

${ }^{4}$ The period length of a $12 \mathrm{~Hz}$ cycle is calculated as 1000 divided by 12 , which is exactly $83.3 \mathrm{~ms}$. 
montage of 62 scalp EEG channels would result in 1953 possible couplings. Two recent studies, however, investigated the role of prestimulus phase coupling on visual perception of a stimulus (Hanslmayr et al., 2007; Kranczioch et al., 2007).

In their study, Hanslmayr et al. (2007) presented one of four possible visual stimuli ( $p, b, d$, or q) for a short duration $(50 \mathrm{~ms}$ ) and the subjects had to indicate which of the four stimuli they perceived. Contrasting trials in which the stimulus was perceived correctly, with trials in which the stimulus was not perceived, Hanslmayr et al. found a pronounced difference in prestimulus alpha phase coupling. Perceived trials exhib ited significantly lower levels of phase coupling between frontal and parietal electrode sites than unperceived trials. Sorting the single trials according to prestimulus alpha phase coupling, the authors showed that phase coupling could account for a large range of the behavioral variance: ranging from $80 \%$ perception performance in the trials with lowest phase coupling, to $40 \%$ perception performance in the trials with highest phase coupling.

Kranczioch et al. (2007) utilized the attentional blink paradigm and investigated the difference between trials in which the second target was perceived correctly and in which the second target was missed. In line with the above cited findings, high levels of prestimulus alpha phase coupling predicted a miss, whereas low levels of phase coupling predicted correct perception of the second target stimulus. Whereas the two studies demonstrated that visual perception performance critically depends on the level of prestimulus alpha phase coupling, no study has yet manipulated the levels of prestimulus phase coupling experimentally.

Taken together, the above cited findings suggest that fluctuations in alpha phase coupling indicate externally and internally oriented states, thus predicting the likelihood of whether a visual stimulus will be perceived or not. Therefore, high levels of alpha phase coupling indicate internally orient ed brain states, whereas low levels of alpha phase coupling indicate externally oriented brain states (Fig. 4C).

\section{Neurophysiological considerations of alpha oscillations}

\subsection{Alpha amplitude and phase at stimulus onset are controlled by thalamo-cortical loops}

One of the main stations of the visual pathway is the lateral geniculate nucleus in the thalamus, in which information from the retina is transferred to the visual cortex by thalamo cortical neurons. Several electrophysiological studies in ani mals revealed that dynamics in thalamo cortical loops generate alpha oscillations in posterior brain regions (Lopes da Silva et al., 1980; Hughes et al., 2004; Hughes and Crunelli, 2007). Moreover, thalamo cortical alpha oscillations gate signal transmission as demonstrated recently by Lorincz et al. (2009) who showed that the firing of thalamo cortical neurons is phase locked to the alpha cycle, with a majority of neurons firing at the negative peak of the alpha cycle. In humans, simultaneous EEG FMRI studies demonstrated that fluctuations in alpha amplitudes are positively correlated with the BOLD signal in the thalamus (Sadaghiani et al., 2010;
Moosmann et al., 2003; Goldman et al., 2002). Moreover, intracranial recordings in human patients suggest that the pathological slowing of the alpha rhythm is due to abnormal thalamo cortical network activity (Sarnthein et al., 2006; Sarnthein and Jeanmonod, 2007).

Together, these findings are consistent with the idea that alpha oscillatory amplitude and phase are generated by thalamo cortical loops. By modulating alpha amplitude and phase at stimulus onset these loops likely gate the bottom up flow of visual information from the thalamus to the cortex. As outlined above, alpha amplitude and phase at stimulus onset are not independent predictors of whether or not a visual stimulus will be perceived. Instead, the predictive role of alpha phase is positively correlated with alpha amplitude. ${ }^{5}$ Thus, alpha amplitude and phase at stimulus onset may both reflect externally oriented or internally oriented brain states at the thalamo cortical level of the visual pathway, though in different time scales. Whereas alpha phase might reflect the fluctuations between these states at fast time scales, alpha power might reflect the more slower fluctuations between internally and externally oriented brain states.

\subsection{Alpha phase-coupling reflects cortico-cortical communication}

Phase coupling between EEG/MEG sensors or brain regions can be regarded as a measure of communication between distant neural assemblies, because neuronal firing patterns are triggered by oscillatory phase (Canolty et al., 2010; Fries, 2005; Varela et al., 2001). Phase synchrony in the alpha frequency band in particular has been shown to reflect the top down flow of information between cortical brain regions in cats (von Stein et al., 2000). In line with this result, a number of human EEG/MEG studies reported an increase in alpha phase coupling during complex cognitive tasks, such as manipulation or maintenance of information in working memory (Sauseng et al., 2005; Palva et al., 2010), or perception of meaningful objects (Freunberger et al., 2008; Mima et al., 2001). Using sophisticated source localization methods Palva et al. (2010) demonstrated that alpha phase coupling between fronto parietal brain regions was parametrically modulated by visual working memory load and predicted individual working memory capacity. Such between subject variations in working memory capacity have recently been shown to reflect successful filtering of relevant from irrelevant items during encoding and maintenance (Vogel et al., 2005). In visual perception paradigms, alpha phase coupling between EEG sensors has been shown to index the perception of a meaningful object, which has been interpreted to reflect the integration of information into the semantic memory system (Freunberger et al., 2008).

Together, these studies strongly suggest that phase coupling in the alpha frequency range may reflect top down flow of information between cortical neural assemblies. Thereby, alpha phase coupling likely indicates the action of higher order internal cognitive processes such as required

\footnotetext{
${ }^{5}$ Note, however, that this effect can also arise from a higher signal to noise ratio in those trials with high alpha power.
} 
during working memory or the perception of meaningful stimuli, as required by the $A B$ task.

\section{Explaining the attentional blink by alpha oscillations}

To provide an account of the attentional blink in terms of alpha oscillations we begin with the assumption that in the typical $A B$ paradigm, the series of events preceding $\mathrm{T} 2$ presentation, induces a brain state which is quite unfavorable for visual perception. Note, however, that the first target is also preceded by some of these events but is still much better perceived than T2, a point to which we will return below. Specifically, we contend that the AB paradigm influences all three parameters of alpha oscillatory activity such that they are maximally in their least favorable (i.e., internally oriented) state at the time the second target occurs. We unpack this argument as follows.

\subsection{Alpha amplitudes}

In the $A B$ paradigm stimuli are usually presented at $10 \mathrm{~Hz}$ (ranging from 7 to $13 \mathrm{~Hz}$ ). Stimulation at this frequency is known to induce steady state visual evoked potentials (Vialatte et al., 2010), which increase alpha amplitude at occipital brain regions. Although presently it is unclear whether such a stimulation induces neural activity that adds onto the ongoing intrinsic alpha oscillation, or whether it enhances alpha amplitudes by driving the intrinsic alpha oscillatory activity (Moratti et al., 2007), there is no doubt that such stimulation effectively enhances alpha amplitude over visual processing regions. Previous studies demonstrated that sSVEPs increase in amplitude when attention is directed toward the stimulus stream (e.g. Müller and Hübner, 2002), which seems to be at odds with our argument that increased alpha power (e.g. due to ssVEPs) reflects an internally oriented processing mode. However, alpha oscillations might be in a resonant state during the $\mathrm{AB}$ task, as they are crucial for performing the task. Therefore, the external stimulus might lead to an increase of (internal) alpha power. As explicated above (Fig. 4A), this creates an unfavorable scenario for the perception of a visual stimulus.

\subsection{Alpha phase at stimulus onset}

By entraining the visual system in the alpha frequency range, the $A B$ paradigm induces phase consistency over repeated trials, which can be seen in the ERP (Fig. 5). In Fig. 5 an example of an ERP dataset (averaged over 17 subjects) from our laboratory is shown for an occipital electrode $(\mathrm{Oz})$ during a $10 \mathrm{~Hz}$ flickering stimulus. The data reveals strong phase locking to the stimulation frequency, as evident in the unfiltered ERP. Alpha oscillatory activity is entrained to the flickering stimulus such that the visual stimuli (squares) are presented exactly at the negative peak. As described above, this resembles an internally oriented brain state, which correlates with poor perceptual performance.

Thus, by presenting the stimuli at a regular frequency around $10 \mathrm{~Hz}$ the $\mathrm{AB}$ paradigm drives alpha amplitude and alpha phase at stimulus onset toward an internally oriented brain state, creating an unfavorable neural environment for the second target. Evidence to support this assumption comes from a recent behavioral study by Martin et al. (2011) who

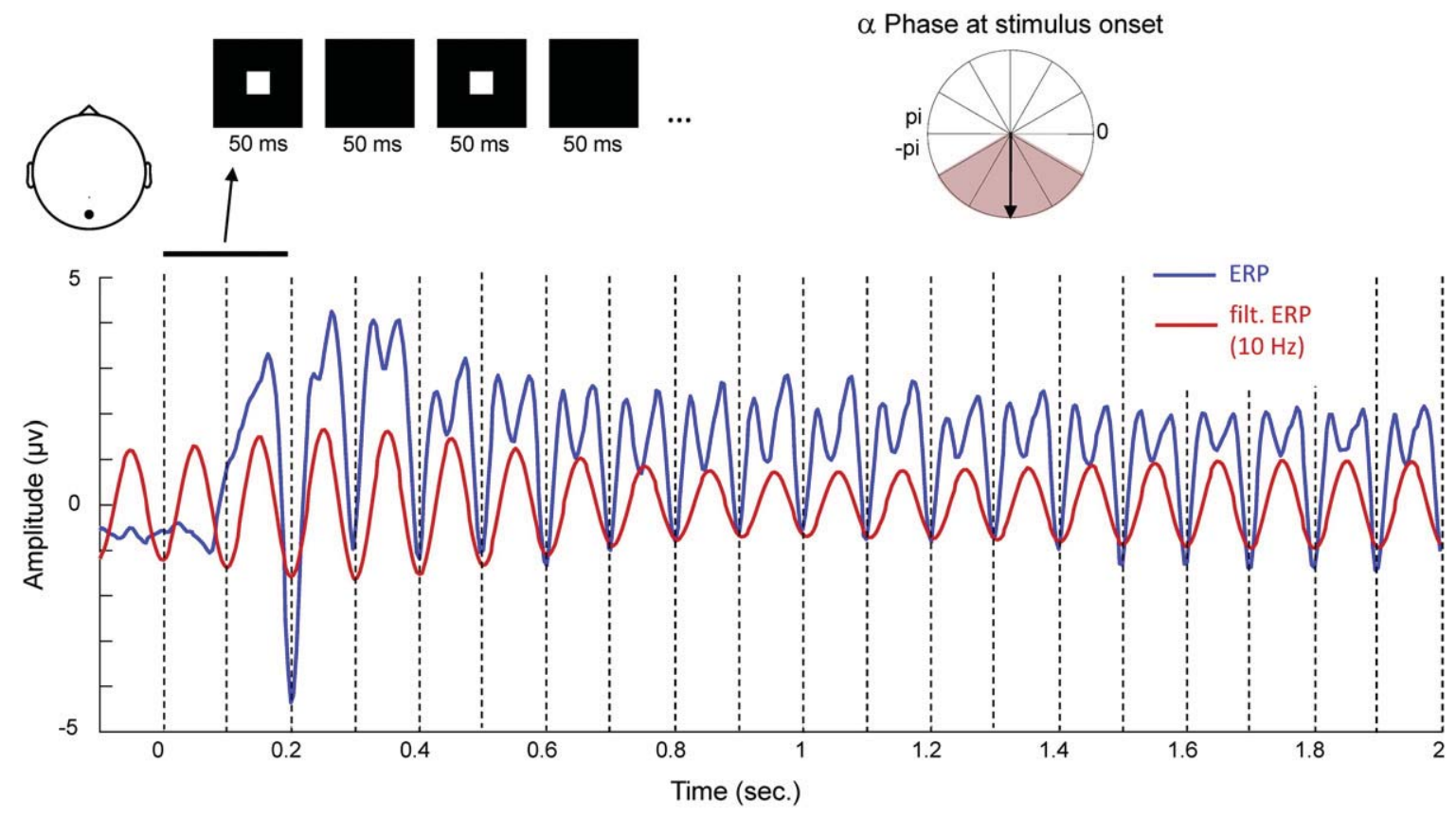

Fig. 5 - The ERP data (electrode Oz; averaged over 17 participants) is shown during a steady-state visual potential experiment. A stimulus (square) flickering at $10 \mathrm{~Hz}$ was presented. The blue line shows the unfiltered ERP, and the red line shows the bandpass-filtered ERP $(8-12 \mathrm{~Hz})$. Note, the constant negative phase at stimulus onset. 
demonstrated that the $\mathrm{AB}$ effect can virtually be abolished by presenting the pretarget stimulus stream irregularly (using a random interstimulus interval). As such an irregular stimula tion does not entrain alpha amplitude, nor does it induce phase consistency at stimulus onset, the results suggest that driving posterior brain regions in a $10 \mathrm{~Hz}$ frequency range is critical to obtain the $\mathrm{AB}$.

Alpha amplitude and phase at stimulus onset, however, cannot alone explain why the $A B$ occurs for the following two reasons: (i) Using a simplified version of the $A B$ paradigm Duncan et al. (1994) presented only two targets successively, each masked, and separated by the same SOAs normally used to index the $A B$. Despite the fact that no pretarget stimulus stream was presented, the results revealed a prominent $A B$ for the second target if presented around $250 \mathrm{~ms}$ after the first target. These results demonstrate that the induction of alpha evoked activity preceding the target presentation is not required for obtaining the basic pattern of the $A B$. (ii) In typical $A B$ experiments 8 to 15 distracter items are presented before the first target. Alpha amplitude and phase at stimulus onset can thus be expected to resemble an internally oriented brain state already at the time the first target is shown. The first target, however, is usually perceived well, in $90 \%$ of the trials. ${ }^{6}$ Therefore, a third process, probably triggered by the perception of the first target must come into play to explain why the $A B$ occurs.

\subsection{Alpha phase-coupling}

Phase coupling in the alpha frequency range between frontal and parietal brain regions is typically triggered by complex cognitive processes such as working memory, or perception of a meaningful stimulus. In the $A B$ paradigm perception of the first target likely relies on such processes. As outlined in section two, successful identification of a target stimulus requires the match between a template held in working memory and between the representation in posterior visual processing regions (Enns and Di Lollo, 2000). Because the target is quickly followed by a mask, this match has to be made internally within the working memory system. Thus, the perception of the first target likely induces an increase in cortico cortical communication as reflected by alpha phase coupling, which drives the brain toward an internally oriented state at the time the second target is presented. Indeed, Mima et al. (2001) showed that successful identification of a visual stimulus triggers increased alpha phase coupling peaking at $250 \mathrm{~ms}$, which perfectly mirrors the time course of the $A B$ (Fig. 2B). As previously suggested, it is an externally, not internally, oriented brain state that is optimal for detection/ identification of a sensory stimulus, thus conditions are in effect to bring about an attentional blink.

\footnotetext{
${ }^{6}$ In the study by Martin et al. (2011) perception of the first target was reduced in the condition in which the pretarget stimuli were presented at a regular rate, compared to the condition in which an irregular stimulation frequency was used. These results further corroborate our assumption that inducing alpha oscilla tory activity via ssVEPs drives the brain toward an internally oriented state.
}

\section{Conclusions}

Integrating recent studies, which reveal a strong link between ongoing alpha activity and perceptual performance, the current review suggests that the $A B$ outcome can well be predicted by alpha oscillations. In particular, the rapid serial visual presentation aspect of the $A B$ paradigm as the brain struggles with the first target drives alpha amplitude, phase at stimulus onset, and phase coupling toward an internally oriented state, which makes it difficult for briefly presented stimuli to be perceived. Although performance for the second target is markedly reduced, it is important to note an $A B$ is obtained in only about $50 \%$ of the trials, ${ }^{7}$ revealing the stochastic nature of the underlying brain processes creating the $A B$. However, the question of why an $A B$ is obtained in some trials and not on other trials is not yet resolved. The currently proposed framework suggests that by combining the three different parameters of alpha oscillatory activity the occurrence of an $\mathrm{AB}$ can be predicted on a single trial level.

An intriguing remaining question is whether some param eters of alpha predict the occurrence of the $A B$ better than others. Based on the above described studies, two hypotheses could be supported: (i) Alpha phase coupling is more critical than alpha power and phase. This hypothesis can be supported by the behavioral study by Duncan et al. (1994) which suggests that alpha amplitude and phase at stimulus onset alone cannot explain why the $A B$ occurs. Moreover, phase coupling has been shown to predict with very high accuracy whether or not a briefly presented visual stimulus is perceived (Hanslmayr et al., 2007). Assuming that alpha amplitude and phase at stimulus onset reflect thalamo cortical activity, and that alpha phase coupling reflects cortico cortical communication, this hypothesis would be consistent with the finding that cortical processing is much more driven by cortico cortical loops, than by thalamo cortical loops ${ }^{8}$ (Douglas and Martin, 2007). (ii) The second hypothesis is that alpha power and phase are more critical than alpha phase coupling. Importantly, the first hypothesis assumes that alpha power and phase are not influenced by the cognitive processes triggered by the presentation of the first target. As no study has yet examined how the processing of the first target affects alpha power and phase consistency, we cannot rule out the possibility that the processing of $\mathrm{T} 1$ drives these two parameters even more strongly toward an internally oriented processing state. These hypotheses can be tested by future studies, hopefully shedding light onto the unresolved issues.

\section{Acknowledgments}

The authors would like to thank Nathan Weisz and Maria Wimber for their very insightful comments on previous

\footnotetext{
${ }^{7}$ In $\mathrm{AB}$ experiments the false alarm rate is typically quite low (around 10\%) which indicates that the subjects correctly perceive the second target in about half of the trials.

${ }^{8}$ Only $1020 \%$ of the input layers in the cortex are thalamocor tical, with the rest originating from other cortical pyramidal cells.
} 
versions of the manuscript. Additionally, the authors thank Maria Wimber for contributing the data presented in Fig. 5.

\section{R EF E R E N C E S}

Bowman, H., Wyble, B., 2007. The simultaneous type, serial token model of temporal attention and working memory. Psychol. Rev. 114, 3870.

Busch, N.A., Dubois, J., VanRullen, R., 2009. The phase of ongoing EEG oscillations predicts visual perception. J. Neurosci. 29 (24), 78697876

Busch, N.A., VanRullen, R., 2010. Spontaneous EEG oscillations reveal periodic sampling of visual attention. Proc. Natl. Acad. Sci. U. S. A. 107, 1604816053.

Buzsáki, G., Draguhn, A., 2004. Neuronal oscillations in cortical networks. Science 304, 19261929.

Callaway, E., Yeager, C.L., 1960. Relationship between reaction time and electroencephalographic alpha phase. Science 132, 17651766.

Canolty, R.T., Ganguly, K., Kennerley, S.W., Cadious, C.F., Koepsell, K., Wallis, J.D., Carmena, J.M., 2010. Oscillatory phase coupling coordinates anatomically dispersed functional cell assemblies. Proc. Natl. Acad. Sci. U. S. A. 1735617361.

Chun, M.M., Potter, M.C., 1995. A two stage model for multiple target detection in rapid serial visual presentation. J. Exp. Psychol. Hum. Percept. Perform. 21, 109127.

Dehaene, S., Sergent, C., Changeux, J.P., 2003. A neuronal network model linking subjective reports and objective physiological data during conscious perception. Proc. Natl. Acad. Sci. U. S. A. 100,85208525

Di Lollo, V., Kawahara, J., Ghorashi, S.M., Enns, J.T., 2005. The attentional blink: resource depletion or temporary loss of control? Psychol. Res. 69, 191200.

Douglas, R.J., Martin, K.A.C., 2007. Mapping the matrix: the ways of neocortex. Neuron 56, 226238.

Duncan, J., Ward, R., Shapiro, K., 1994. Direct measurement of attentional dwell time in human vision. Nature 369, 313315

Dustman, R.E., Beck, E.C., 1965. Phase of alpha brain waves, reaction time and visually evoked potentials. Electroencephalogr. Clin. Neurophysiol. 18, 433440.

Enns, J.T., Di Lollo, V., 2000. What's new in visual masking? Trends Cogn. Sci. 4, 345352.

Ergenoglu, T., Demiralp, T., Bayraktaroglu, Z., Ergen, M., Beydagi, H., Uresin, Y., 2004. Alpha rhythm of the EEG modulates visual detection performance in humans. Brain Res. Cogn. Brain Res. 20, 376383

Freunberger, R., Klimesch, W., Griesmayr, B., Sauseng, P., Gruber, W., 2008. Alpha phase coupling reflects object recognition. Neuroimage 42, 928935.

Fries, P., 2005. A mechanism for cognitive dynamics: neuronal communication through neuronal coherence. Trends Cogn. Sci. 9, 474480 .

Goldman, R.I., Stern, J.M., Engel, J., Cohen, M.S., 2002. Simultaneous EEG and fMRI of the alpha rhythm. Neuroreport 13, 24872492

Gruber, W., Klimesch, W., Sauseng, P., Doppelmayr, M., 2005. Alpha phase synchronization predicts P1 and N1 latency and amplitude size. Cereb. Cortex 15, 371377.

Gross, J., Kujala, J., Hamalainen, M., Timmermann, L., Schnitzler, A., Salmelin, R., 2001. Dynamic imaging of coherent sources: studying neural interactions in the human brain. Proc. Natl. Acad. Sci. U. S. A. 98, 694699.

Gross, J., Schmitz, F., Schnitzler, I., Kessler, K., Shapiro, K., Hommel, B., Schnitzler, A., 2004. Modulation of long range neural synchrony reflects temporal limitations of visual attention in humans. Proc. Natl. Acad. Sci. U. S. A. 101, 1305013055.
Gross, J., Schmitz, F., Schnitzler, I., Kessler, K., Shapiro, K., Hommel, B., Schnitzler, A., 2006. Anticipatory control of long range phase synchronization. Eur. J. Neurosci. 24, 20572060

Hanslmayr, S., Aslan, A., Staudigl, T., Klimesch, W., Herrmann, C.S., Bäuml, K. H., 2007. Prestimulus oscillations predict visual perception performance between and within subjects. Neuroimage 37, 14651473.

Hanslmayr, S., Klimesch, W., Sauseng, P., Gruber, W., Doppelmayr, M., Freunberger, R., Pecherstorfer, T., 2005. Visual discrimination performance is related to decreased alpha amplitude but increased phase locking. Neurosci. Lett. 375, 6468.

Hughes, S.W., Crunelli, V., 2007. Just a phase they're going through: the complex interaction of intrinsic high threshold bursting and gap junctions in the generation of thalamic alpha and theta rhythms. Int. J. Psychophysiol. 64, 317

Hughes, S.W., Lörincz, M., Cope, D.W., Blethyn, K.L., Kékesi, K A., Parri, H.R., Juhász, G., Crunelli, V., 2004. Synchronized oscillations at alpha and theta frequencies in the lateral geniculate nucleus. Neuron 42, 253268.

Jacobs, J., Kahana, M.J., Ekstrom, A.D., Fried, I., 2007. Brain oscillations control timing of single neuron activity in humans. J. Neurosci. 27, 38393844.

Jensen, O., Mazaheri, A., 2010. Shaping functional architecture by oscillatory alpha activity: gating by inhibition. Front. Hum. Neurosci. 4, 186

Jolicoeur, P., 1998. Modulation of the attentional blink by on line response selection: evidence from speeded and unspeeded Task sub 1 decisions. Mem. Cognit. 26, 10141032.

Jolicoeur, P., 1999. Concurrent response selection demands modulate the attentional blink. J. Exp. Psychol. Hum. Percept. Perform. 25, 10971113.

Jolicoeur, P., Dell'Acqua, R., 1998. The demonstration of short term consolidation. Cogn. Psychol. 36, 138202.

Kawahara, J., Enns, J.T., Di Lollo, V., 2006. The attentional blink is not a unitary phenomenon. Psychol. Res. 70, 405413.

Keil, A., Ihssen, N., Heim, S., 2006. Early cortical facilitation for emotionally arousing targets during the attentional blink. BMC Biol. 4, 23.

Keil, A., Heim, S., 2009. Prolonged reduction of electrocortical activity predicts correct performance during rapid serial visual processing. Psychophysiology 46, 718725.

Klimesch, W., Sauseng, P., Hanslmayr, S., 2007. EEG alpha oscillations: the inhibition timing hypothesis. Brain Res. Rev. 53,6388

Kranczioch, C., Debener, S., Maye, A., Engel, A.K., 2007. Temporal dynamics of access to consciousness in the attentional blink. Neuroimage 37, 947955.

Kuhbandner, C., Hanslmayr, S., Maier, M.A., Pekrun, R., Spitzer, B., Pastötter, B., Bäuml, K. H., 2009. Effects of mood on the speed of conscious perception: behavioural and electrophysiological evidence. Soc. Cogn. Affect. Neurosci. 4, 286293.

Lachaux, J.P., Rodriguez, E., Martinerie, J., Varela, F.J., 1999. Measuring phase synchrony in brain signals. Hum. Brain Mapp. 8, 194208

Lakatos, P., Karmos, G., Mehta, A.D., Ulbert, I., Schroeder, C.E., 2008. Entrainment of neuronal oscillations as a mechanism of attentional selection. Science 320, 110113.

Lakatos, P., Shah, A.S., Knuth, K.H., Ulbert, I., Karmos, G., Schroeder, C.E., 2005. An oscillatory hierarchy controlling neuronal excitability and stimulus processing in the auditory cortex. J. Neurophysiol. 94, 19041911.

Lee, H., Simpson, G.V., Logothetis, N.K., Rainer, G., 2005. Phase locking of single neuron activity to theta oscillations during working memory in monkey extrastriate visual cortex. Neuron 45, 147156.

Linkenkaer Hansen, K., Nikouline, V.V., Palva, J.M., Ilmoniemi, R.J., 2001. Long range temporal correlations and scaling behavior in human brain oscillations. J. Neurosci. 21, 13701377. 
Linkenkaer Hansen, K., Nikulin, V.V., Palva, S., Ilmoniemi, R.J., Palva, J.M., 2004. Prestimulus oscillations enhance psychophysical performance in humans. J. Neurosci. 24, 1018610190.

Lopes da Silva, F.H., Vos, J.E., Mooibroek, J., Van Rotterdam, A., 1980. Relative contributions of intracortical and thalamo cortical processes in the generation of alpha rhythms, revealed by partial coherence analysis. Electroencephalogr. Clin. Neurophysiol. 50, 449456

Lopes da Silva, F., 1991. Neural mechanisms underlying brain waves: from neural membranes to networks. Electroencephalogr. Clin. Neurophysiol. 79, 8193.

Luck, S.J., Vogel, E.K., Shapiro, K.L., 1996. Word meanings can be accessed but not reported during the attentional blink. Nature 382, 616618

Lorincz, M.L., Kékesi, K.A., Juhász, G., Crunelli, V., Hughes, S.W., 2009. Temporal framing of thalamic relay mode firing by phasic inhibition during the alpha rhythm. Neuron 63, 683696

Makeig, S., Westerfield, M., Jung, T. P., Enghoff, S., Townsend, J., Courchesne, E., Seinowski, T.J., 2002. Dynamic brain sources of visual evoked responses. Science 295, 690694.

Marois, R., Chun, M.M., Gore, J.C., 2000. Neural correlates of the attentional blink. Neuron 28, 299308.

Marois, R., Yi, D.J., Chun, M.M., 2004. The neural fate of consciously perceived and missed events in the aftentional blink. Neuron 41, 465472.

Martin, E.W., Enns, J.T., Shapiro, K.L., 2011. Turning the Attentional Blink On and Off: opposing Effects of Spatial and Temporal Discontinuity. Psych. Bull. Rev. 18, 295301.

Mathewson, K.E., Fabiani, M., Gratton, G., Beck, D.M., Lleras, A., 2010. Rescuing stimuli from invisibility: inducing a momentary release from visual masking with pre target entrainment. Cognition 115, 186191.

Mathewson, K.E., Gratton, G., Fabiani, M., Beck, D.M., Ro, T., 2009 To see or not to see: prestimulus alpha phase predicts visual awareness. J. Neurosci. 29, 27252732.

Mima, T., Oluwatimilehin, T., Hiraoka, T., Hallett, M., 2001. Transient interhemispheric neuronal synchrony correlates with object recognition. J. Neurosci. 21, 39423948.

Moratti, S., Clementz, B.A., Gao, Y., Ortiz, T., Keil, A., 2007. Neural mechanisms of evoked oscillations: stability and interaction with transient events. Hum. Brain Mapp. 28, 13181333.

Moosmann, M., Ritter, P., Krastel, I., Brink, A., Thees, S., Blankenburg, F., Taskin, B., Obrig, H., Villringer, A., 2003. Correlates of alpha rhythm in functional magnetic resonance imaging and near infrared spectroscopy. Neuroimage 20, 145158

Müller, M.M., Hübner, R., 2002. Can the spotlight of attention be shaped like a doughnut? Evidence from steady state visual evoked potentials. Psychol. Sci. 13, 119124.

Olivers, C.N.L., Meeter, M., 2008. A boost and bounce theory of temporal attention. Psychol. Rev. 115, 836863.

Palva, J.M., Monto, S., Kulashekhar, S., Palva, S., 2010. Neuronal synchrony reveals working memory networks and predicts individual memory capacity. Proc. Natl. Acad. Sci. U. S. A. 107, 75807585.

Pashler, H., 1994. Dual task interference in simple tasks: data and theory. Psychol. Bull. 116, 220244.

Pfurtscheller, G., Aranibar, A., 1977. Event related cortical desynchronization detected by power measurements of scalp EEG. Electroencephalogr. Clin. Neurophysiol. 42, 817826

Phelps, E.A., Ling, S., Carrasco, M., 2006. Emotion facilitates perception and potentiates the perceptual benefits of attention. Psychol. Sci. 17, 292299.

Raymond, J.E., Shapiro, K.L., Arnell, K.M., 1992. Temporary suppression of visual processing in an RSVP task: an attentional blink? J. Exp. Psychol. Hum. Percept. Perform. 18, 849860.

Romei, V., Brodbeck, V., Michel, C., Amedi, A., Pascual Leone, A., Thut, G., 2008. Spontaneous fluctuations in posterior alpha band
EEG activity reflect variability in excitability of human visual areas. Cereb. Cortex 18, 20102018.

Romei, V., Gross, J., Thut, G., 2010. On the role of prestimulus alpha rhythms over occipito parietal areas in visual input regulation: correlation or causation? J. Neurosci. 30, 86928697.

Sadaghiani, S., Scheeringa, R., Lehongre, K., Morillon, B., Giraud, A. L., Kleinschmidt, A., 2010. Intrinsic connectivity networks, alpha oscillations, and tonic alertness: a simultaneous electroencephalography/functional magnetic resonance imaging study. J. Neurosci. 30, 1024310250.

Sarnthein, J., Jeanmonod, D., 2007. High thalamocortical theta coherence in patients with Parkinson's disease. J. Neurosci. 27, 124131

Sarnthein, J., Stern, J., Aufenberg, C., Rousson, V., Jeanmonod, D., 2006. Increased EEG power and slowed dominant frequency in patients with neurogenic pain. Brain 129, 5564.

Sauseng, P., Klimesch, W., Doppelmayr, M., Pecherstorfer, T., Freunberger, R., Hanslmayr, S., 2005. EEG alpha synchronization and functional coupling during top down processing in a working memory task. Hum. Brain Mapp. 26, 148155.

Schubert, R., Haufe, S., Blankenburg, F., Villringer, A., Curio, G., 2009. Now you'll feel it, now you won't: EEG rhythms predict the effectiveness of perceptual masking. J. Cogn. Neurosci. 21, 24072419.

Shapiro, K.L., Arnell, K.M., Raymond, J.E., 1997. The attentional blink. Trends Cogn. Sci. 1, 291296.

Shapiro, K.L., Raymond, J.E., Arnell, K.M., 1994. Attention to visual pattern information produces the attentional blink in rapid serial visual presentation. J. Exp. Psychol. Hum. Percept. Perform. 20, 357371

Shapiro, K.L., Johnston, S.J., Vogels, W., Zaman, A., Roberts, N., 2007. Increased functional magnetic resonance imaging activity during nonconscious perception in the attentional blink. Neuroreport 18, 341345.

Slagter, H.A., Lutz, A., Greischar, L.L., Niewenhuis, S., Davidons, R.J., 2009. Theta phase synchrony and conscious target perception: impact of intense mental training. J. Cogn. Neurosci. 21, 15361549.

von Stein, A., Chiang, C., König, P., 2000. Top down processing mediated by interareal synchronization. Proc. Natl. Acad. Sci. U. S. A. 97, 1474814753

von Stein, A., Sarnthein, J., 2000. Different frequencies for different scales of cortical integration: from local gamma to long range alpha/theta synchronization. Int. J. Psychophysiol. 38, 301313.

Tallon Baudry, C., Bertrand, O., Delpuech, C., Pernier, J., 1996. Stimulus specificity of phase locked and non phase locked $40 \mathrm{~Hz}$ visual responses in human. J. Neurosci. 16, 42404249

van Dijk, H., Schoffelen, J. M., Oostenveld, R., Jensen, O., 2008. Prestimulus oscillatory activity in the alpha band predicts visual discrimination ability. J. Neurosci. 28, 18161823.

VanRullen, R., Reddy, L., Koch, C., 2006. The continuous wagon wheel illusion is associated with changes in electroencephalogram power at approximately $13 \mathrm{~Hz}$. J. Neurosci. 26, 502507.

Varela, F., Lachaux, J.P., Rodriguez, E., Martinerie, J., 2001. The brainweb: phase synchronization and large scale integration. Nat. Rev. Neurosci. 2, 229239

Varela, F.J., Toro, A., John, E.R., Schwartz, E.L., 1981. Perceptual framing and cortical alpha rhythm. Neuropsychologia 19, 675686.

Vialatte, F. B., Maurice, M., Dauwels, J., Cichocki, A., 2010. Steady state visually evoked potentials: focus on essential paradigms and future perspectives. Prog. Neurobiol. 90, 418438

Vogel, E.K., McCollough, A.W., Machizawa, M.G., 2005. Neural measures reveal individual differences in controlling access to working memory. Nature 438, 500503.

Ward, R., Duncan, J., Shapiro, K., 1996. The slow time course of visual attention. Cogn. Psychol. 30, 79109. 